Ann. Biol. anim. Bioch. Biophys., 1974, 14 (I), 67-85.

\title{
ÉTUDE ÉLECTROMYOGRAPHIQUE DE LA MOTRICITÉ DE L'UTÉRUS CHEZ LA BREBIS. ACTION DES HORMONES
}

\author{
J.-P. ROUSSEAU et Marie-Jeanne PRUD'HOMME \\ Laboratoire de Neurophysiologie végétative, SN 4, Université de Lille I, \\ 59650 Villeneuve d'Ascq \\ Station de Physiologie de la Reproduction. \\ Centre de Recherches de Tours, I. N. R. A., \\ B. P. 1 Nouzilly, 37380 Monnaie
}

\section{RÉSUMÉ}

Nous avons enregistré in vivo l'activité électrique et mécanique du myomètre chez 46 brebis, les unes étant castrées et traitées aux cestrogènes ou à la progestérone, les autres étant cycliques. L'activité électrique a été recueillie dans les conditions expérimentales aiguës, soit par des micropipettes de verre extracellulaires remplies de $\mathrm{KCl} 3 \mathrm{M}$, soit par des électrodes métalliques externes posées sur la séreuse. Dans les conditions expérimentales chroniques, nous avons placé à demeure sur l'utérus des électrodes de platine. L'activité mécanique a été appréciée par les variations de la pression intra-utérine ou les variations locales de la tension musculaire.

De l'ensemble des résultats, il ressort que :

I. Chez la brebis castrée, les oestrogènes induisent une activité électromyographique (EMG) rythmique constituée par des salves de potentiels d'action liées aux variations de la pression intra-utérine. Cette activité EMG est maximale 24 à 36 heures après le traitement au benzoate d'œestradiol, ce qui coïncide avec le début de l'œestrus comportemental.

Chez la brebis castrée, traitée à la progestérone, l'activité EMG de l'utérus est constituée par des variations lentes de potentiel qui ne sont pas synchronisées avec les contractions du myomètre.

Chez la brebis castrée, sous imprégnation œestrogénique, des doses faibles d'ocytocine provoquent une décharge continue de potentiels d'action. Des doses élevées font disparaître les salves de potentiels, alors que le myomètre est en état de contracture.

2. Chez la brebis cyclique, au cours de l'œestrus, l'utérus présente une activité EMG rythmique spontanée identique à celle enregistrée chez la brebis castrée en œestrus induit. A part quelques brebis chez lesquelles des salves ont été enregistrées le $6^{\mathrm{e}}$ jour du cycle, la seule période au cours de laquelle l'activité EMG de l'utérus est caractérisée par des salves de potentiels d'action, se

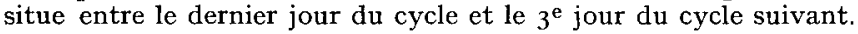

3. Chez la brebis éveillée, castrée, en cestrus induit ou cyclique, en début d'œstrus, des stimulations vaginales (distension ou stimulation superficielle du vagin) provoquent une nette augmentation de l'activité électrique du myomètre. Les mêmes stimulations sont sans effet chez la brebis anesthésiée. 


\section{INTRODUCTION}

La motricité utérine a été étudiée in vivo surtout chez la Femme au cours de l'accouchement (Csapo et TAKedA, I963; EFFER et al., I969; KrapoHL, Myers et CALde Yro-Barcia, I970; Wolfs et RotTinghuis, I970) ou in vitro chez la Ratte et la Lapine, dans le cadre de recherches sur la physiologie élémentaire de la fibre musculaire lisse (MARShali, I959; KURIYAMa et CsAPo, I961 ; CSAPO, I962 ; MARSHALI, I962). Ce sont d'ailleurs ces dernières qui ont permis de mieux connaître l'action des hormones sexuelles sur l'activité électrique et mécanique du myomètre, et leurs effets sur le niveau d'excitabilité de la cellule musculaire et la propagation de la contraction.

Chez la Brebis, nous possédons par contre peu de données, toutes obtenues par manométrie, dans des conditions bien précises telles que la parturition (HIndson, SCHOFIELd et TURNER, I968) ou l'œstrus (MANN, I969; LiGHTFOoT, I970). Les études soulignent principalement les modifications de l'amplitude et de la fréquence des contractions utérines, consécutives à la mise en place de dispositifs intra-utérins, à l'accouplement ou à l'injection intraveineuse d'ocytocine, mais ne mettent pas l'accent sur l'influence des hormones sexuelles. Seuls les travaux de BRINSFIEID (I968), sur des lambeaux d'utérus en survie in vitro, montrent que sous imprégnation de progestérone, les contractions utérines sont moins amples et moins fréquentes que sous imprégnation œstrogénique. Aussi, nous a-t-il semblé souhaitable de compléter ces résultats obtenus in vitro par des expériences effectuées in vivo. Nous avons donc enregistré l'activité électromyographique (EMG) de l'utérus chez la Brebis, afin de préciser l'action des hormones sexuelles et de l'ocytocine sur le myomètre de cette espèce.

Notre étude a été réalisée chez la brebis castrée traitée par des hormones (œstrogènes, progestérone, ocytocine) et chez la brebis cyclique à différents moments de son cycle œstrien. Nous avons également enregistré les effets des stimulations vaginales sur la motricité utérine, chez la brebis en période d'œstrus.

\section{TECHNIQUE}

Les expériences sont réalisées dans les conditions aiguës ou chroniques et portent sur 46 brebis de race Préalpes ou Solognote, castrées ou cycliques.

Les brebis castrées sont soumises à différents traitements hormonaux. L'cestrus est induit chez les unes soit par le traitement de RoBinson (I962) : progestérone plus œestrogènes, soit par une seule injection de $50 \mu \mathrm{g}$ de benzoate d'œstradiol. Les autres reçoivent $25 \mathrm{mg}$ de progestérone par jour pendant 6 jours.

La détection de l'ostrus comportemental (induit ou non) est effectuée dans tous les cas par un bélier; chez les brebis cycliques, elle permet de dater les cycles dont la durée est de I6 à I 7 jours.

$$
\text { I. - Conditions expérimentales aiguës }
$$

Les expériences de ce type sont effectuées sur des brebis castrées et traitées. Elles sont pratiquées, soit le jour même de l'œstrus induit, c'est-à-dire 24 à $4^{8}$ heures après le traitement hormonal défini précédemment, soit systématiquement 24 heures après la dernière injection de progestérone. 
L'animal est anesthésié par une solution de barbituriques $(0,5 \mathrm{~g}$ de pentothal et $0,2 \mathrm{~g}$ de nembutal dissous dans $25 \mathrm{ml}$ d'eau distillée) injectée dans la veine jugulaire. L'anesthésie est ensuite entretenue par l'inhalation d'un mélange gazeux d'oxygène et de fluothane. La brebis étant placée en décubitus dorsal, on pénètre dans la cavité abdominale par une incision de I $2 \mathrm{~cm}$ pratiquée sur la ligne médiane, en avant de la mamelle. L'utérus est extériorisé et maintenu

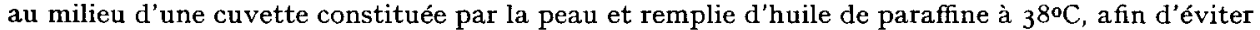
la dessication et le refroidissement de l'organe. On peut alors enregistrer l'activité électrique du myomètre pendant 6 à Io heures consécutives, dans des conditions monopolaires, à l'aide de micropipettes de verre extracellulaires remplies de $\mathrm{KCl} 3 \mathrm{M}$, utilisées en montage flottant selon la technique de Woodbury et Brady (I956), ou par des électrodes constituées d'un seul fil de cuivre spiralé, à l'extrémité duquel une couche de noir de platine est déposée selon la méthode de Kohlrausch (in Nastuk, I963). Mais nous avons utilisé le plus couramment des électrodes métalliques externes bipolaires posées sur la séreuse, formées d'une torsade de 2 fils de cuivre émaillés et dénudés à leur extrémité.

Les variations de la pression intra-utérine sont recueillies par un cathéter rempli de solution physiologique, ou par un ballonnet gonflé de 2 à $5 \mathrm{ml}$ d'air, placés dans la lumière de l'utérus. Pour enregistrer les contractions localisées du myomètre, nous insérons un microballonnet rempli d'eau dans l'épaisseur de la paroi musculaire, selon la technique de Auffray, MARTINET et RÉrat (I967).

\section{2. - Conditions expérimentales chroniques}

Nous avons utilisé des brebis castrées ou cycliques. Les animaux sont anesthésiés dans les mêmes conditions que précédemment et placés en décubitus dorsal. L'utérus est extériorisé grâce à une incision ventrale et les électrodes sont suturées sur la séreuse par 3 points de soie (aiguilles serties). Ces électrodes placées à demeure sont constituées de 2 fils de platine de $0,5 \mathrm{~mm}$ de diamètre et de $I, 5 \mathrm{~mm}$ de longueur, solidaires d'un socle de résine acrylique et reliés à des fils conducteurs gainés de téflon (Rousseau, 1970). Affinées, les extrémités de ces pointes de platine sont d'environ $200 \mu \mathrm{m}$ et sont distantes de $2 \mathrm{~mm}$ (fig. I). Chez les brebis cycliques, un microballonnet est inséré dans la musculeuse, au voisinage de l'implantation des électrodes, afin de recueillir l'activité mécanique localisée du myomètre.

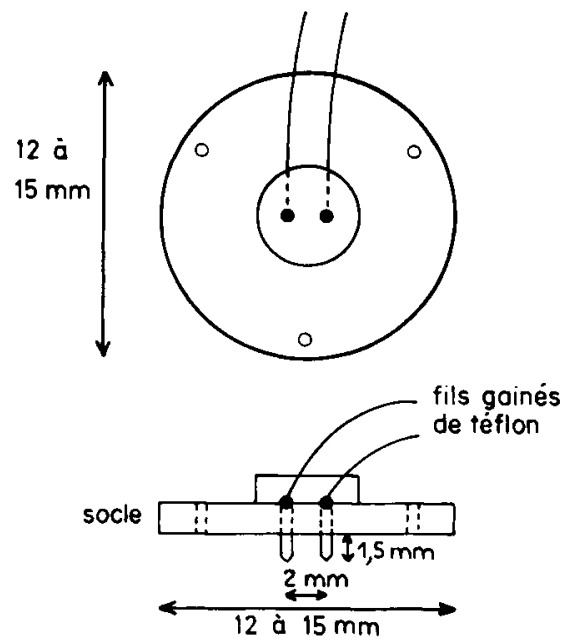

FIG. I. - Electrodes de réception : vue de dessus et vue de profil

Les électrodes sont constituées de deux fils de platine, solidaires d'un socle de résine acrylique, soudés à des fils conducteurs gainés de téflon. Le socle est fixé sur la paroi externe de l'utérus par 3 points de suture.

Les fils conducteurs et le cathéter du microballonnet sont rassemblés. Nous les faisons sortir de la cavité abdominale par l'incision ventrale et cheminer sous la peau jusque sur le dos. Ils y sont récupérés par une incision cutanée, en regard des dernières vertèbres dorsales. L'ouverture initiale est refermée plan par plan. L'animal est alors placé en décubitus sterno-abdominal, la tête prenant appui sur un support fixe. Les fils conducteurs sont soudés aux broches d'un connecteur femelle, les points de contact étant isolés les uns des autres et du tissu environnant 
par une résine (PALAKos) parfaitement tolérée. L'ensemble est maintenu par des points à la peau du dos, de telle sorte que le connecteur affleure juste la surface cutanée et ne détermine aucune aspérité. L'extrémité du cathéter est également fixée sur le dos.

Les brebis cycliques et les brebis castrées, alors traitées selon les modalités définies précédemment, peuvent être utilisées pendant 3 à 6 semaines.

\section{3. - Enregistrement}

Les variations de pression obtenues sont transformées en différence de potentiel grâce à un capteur électronique de type inductif. L'électromyogramme et le mécanogramme sont enregistrés sur papier grâce à un inscripteur à plumes classique ou à l'aide d'un oscilloscope dont l'écran est photographié par une caméra.

\section{RÉSULTATS}

Afin de mieux appréhender et d'interpréter les modifications de l'activité électrique du myomètre chez la brebis, au cours de son cycle sexuel ou sous l'influence de stimulations vaginales, il nous a semblé utile d'enregistrer au préalable l'activité du muscle utérin dans des conditions expérimentales aiguës ou chroniques, chez des brebis castrées, soumises à différents traitements hormonaux.

\section{A. - Étude chez l'animal castré anesthésié}

I. Action des cestrogènes.

Chez la brebis castrée, anesthésiée dès les premières heures de l'œstrus induit, soit 24 heures après l'injection d'œstrogènes, nous enregistrons en un point de la
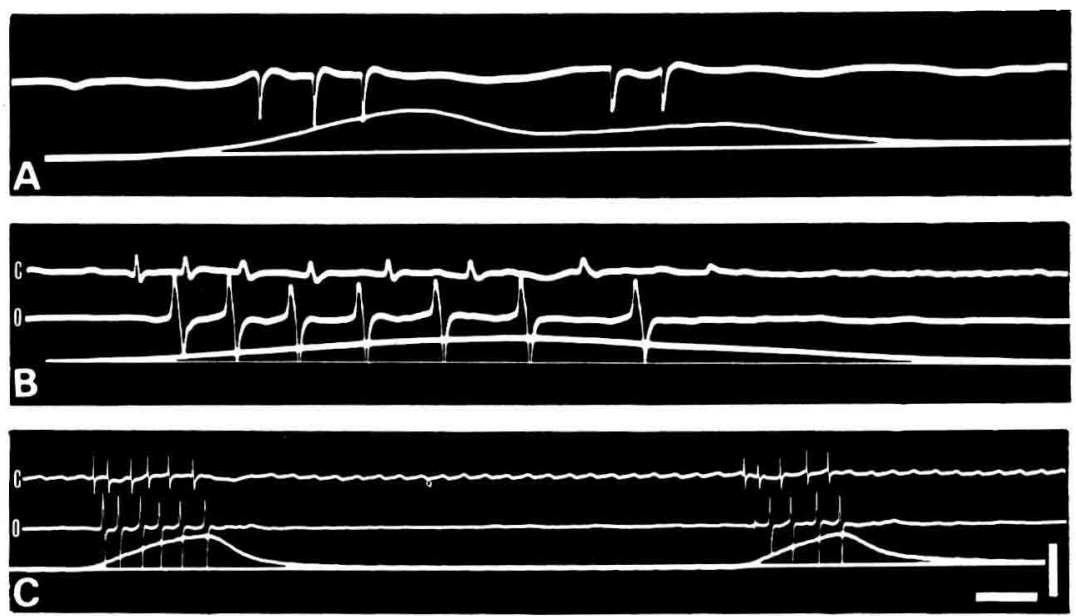

FIG. 2. - Enregistrements de l'activité du myomètre par différents types d'électrodes, chez une brebis castrée, anesthésiée 24 heures après l'injection de benzoate d'œestradiol

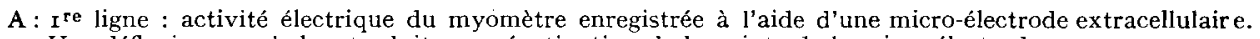
Une déflexion vers le bas traduit une négativation de la pointe de la micro-électrode. $2^{\mathrm{e}}$ ligne: variations de la pression intra-utérine.

$\mathrm{B}$ : Activité électrique du myomètre enregistrée à l'aide d'électrodes bipolaires placées au niveau des extrémités cervicale (c) et ovarienne (o) d'une même corne. Les salves sont contemporaines de l'augmentation de la pression intra-utérine dans la corne considérée ( 3 e ligne).

C : Mêmes enregistrements qu'en $B$, à une vitesse d'enregistrement plus faible. Etalonnages : amplitude : $2 \mathrm{mV}(\mathrm{A}), 500 \mu \mathrm{V}(\mathrm{B}, \mathrm{C})$ temps : I s (A, B), 4 s (C) 
corne utérine, $\mathrm{I}, 7$ à 2 salves de potentiels par minute. Chaque salve dure 4 à Io secondes. Elle comprend de 4 à Io potentiels d'action dont la durée est d'environ I5O à $250 \mathrm{~ms}$ et dont la fréquence moyenne varie de 0,6 à $\mathrm{x}, 5$ potentiel par seconde (fig. 2). La fréquence instantanée, maximale dès le début de la salve décroît rapidement (fig. 3).
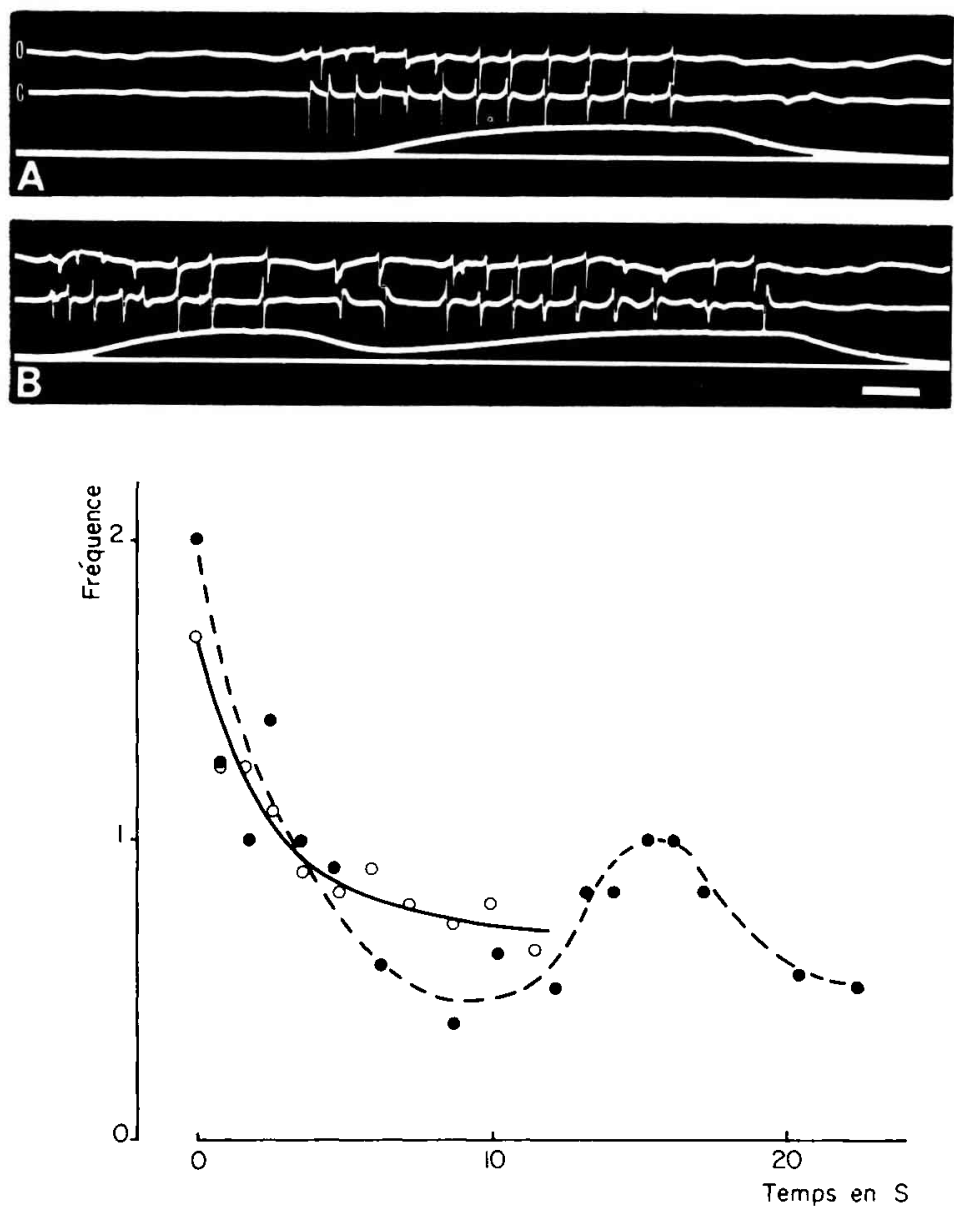

Fig. 3. - Enregistrements de l'activité du myomètre

chez une brebis castrée, anesthésiée 24 heures après l'injection de benzoate d'cstradiol

L'EMG est enregistré à l'aide d'électrodes bipolaires placées au niveau des extrémités ovarienne (o) et cervicale (c) d'une même corne. Les salves sont contemporaines de l'augmentation de la pression intrautérine dans la corne considérée ( $3^{\mathrm{e}}$ ligne).

Le graphique représente les variations de la fréquence par seconde des potentiels constituant une salve lors de 2 contractions successives du myometre. Les courbes en trait plein, et en pointillé ont été obtenues respectivement à partir des enregistrements $\mathrm{A}$ et $\mathrm{B}$.

$$
\text { Étalonnage : temps : } 2 \text { s (A et B) }
$$

La forme et l'amplitude des potentiels varient selon la méthode de réception. Ceux recueillis à l'aide d'une micro-électrode extracellulaire sont négatifs, monophasiques, de $2 \mathrm{mV}$ d'amplitude (fig. $2 \mathrm{~A}$ ). En dérivation bipolaire, ils sont d'amplitude moindre $(0,25$ à $\mathrm{I}, 2 \mathrm{mV})$ et le plus souvent d'allure biphasique (fig. $2 \mathrm{C}$ ). 
L'activité mécanique se traduit par d'amples variations de la pression intrautérine de 20 à $25 \mathrm{~mm}$ de $\mathrm{Hg}$ (fig. $4 \mathrm{~A}$ et $5 \mathrm{~A}$ ). Elle est étroitement liée à l'activité électrique, puisqu'une salve de potentiels apparaît toujours lors de la phase active d'une contraction (fig. 2 et 3 ). Plus la durée de la salve est grande, plus la contraction est prolongée (fig. 3) et l'apparition d'un ou deux potentiels entre deux salves carac-
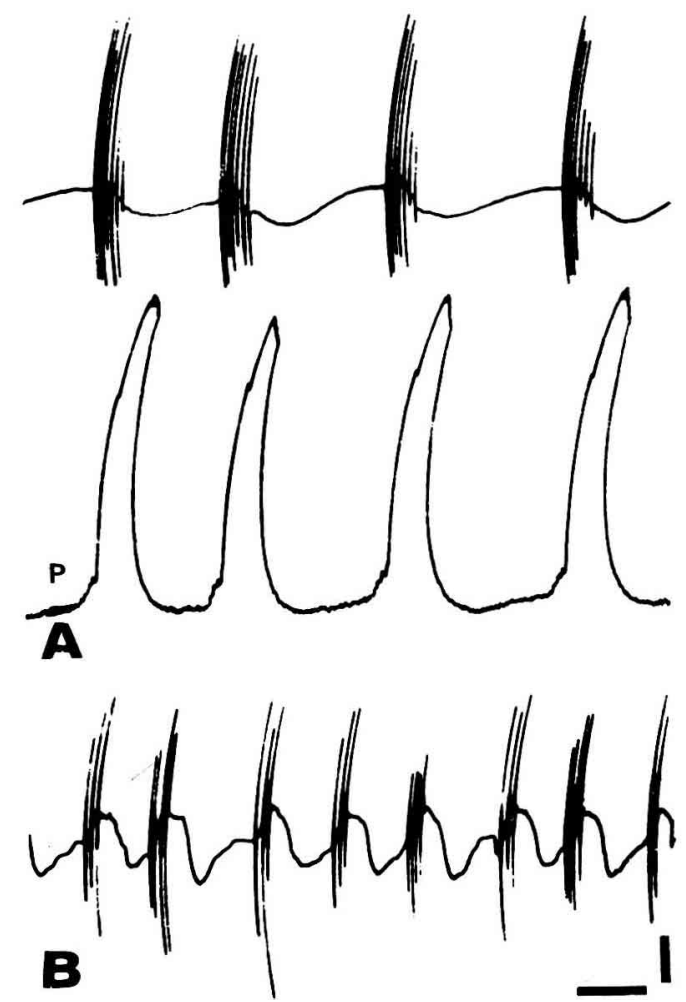

FIG. 4. - Enregistrements de l'activité rythmique de l'utérus chez deux brebis castrées traitées aux cestrogènes

A : EMG et pression intra-utérine (P) enregistrés chez une brebis anesthésiée au fluothane.

$\mathrm{B}: \mathrm{EMG}$ recueilli chez l'autre brebis éveillée.

Le rythme des salves est 2 fois plus rapide chez la brebis éveillée que chez la brebis anesthésiée, le moment de l'enregistrement par rapport à celui de l'injection d'œstrogènes étant identique duns les deux expériences.

Etalonnages : amplitude : Ioo $\mu \mathrm{V}(\mathrm{A}), 500 \mu \mathrm{V}$ (B)
temps : $20 \mathrm{~s}$

téristiques est régulièrement concomitante d'une faible variation de pression de l'ordre de $5 \mathrm{~mm} \mathrm{Hg}$ (fig. 6). Cependant, les rapports temporels entre les deux types d'activité varient selon la méthode utilisée pour l'enregistrement des pressions. Le début de la salve peut précéder de 2 secondes ou suivre d'une seconde le début de l'augmentation de la pression endoluminale. Par contre, lorsque nous recueillons plus localement l'activité mécanique du myomètre, grâce à un microballonnet, la salve enregistrée au même endroit précède toujours de 0,5 seconde l'augmentation de la pression. 

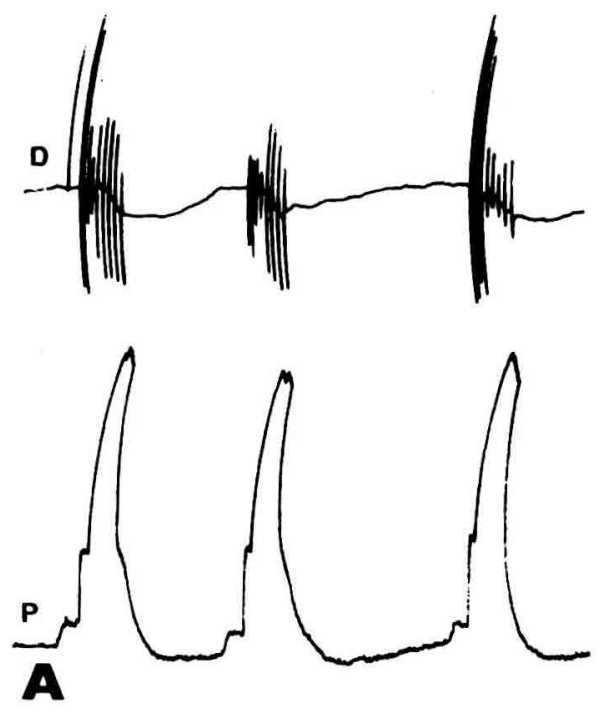

FIG. 5. -- Activité du myomètre enregistrée à l'aide d'électrodes bipolaires, chez deux brebis castrées, anesthésiées

A : Activité rythmique enregistrée 24 heures après l'injection d'œestrogènes.

$$
\begin{aligned}
& \text { D : EMG de la corne droite } \\
& \mathrm{P}: \text { pression intra-utérine }
\end{aligned}
$$

B : Activité enregistrée 24 heures après la fin du traitement à la progestérone.

$$
\begin{aligned}
& G \text { : EMG de la corne gauche }(G) \\
& D: \text { EMG de la corne droite (D) } \\
& P \text { : pression intra-utérine (P) }
\end{aligned}
$$

Noter les différences d'activités électrique et mécanique recueillies chez ces deux animaux.

Étalonnages : amplitude : roo $\mu \mathrm{V}, 5 \mathrm{~mm} \mathrm{Hg}(\mathrm{P})$ temps ; I5 $\mathrm{s}$
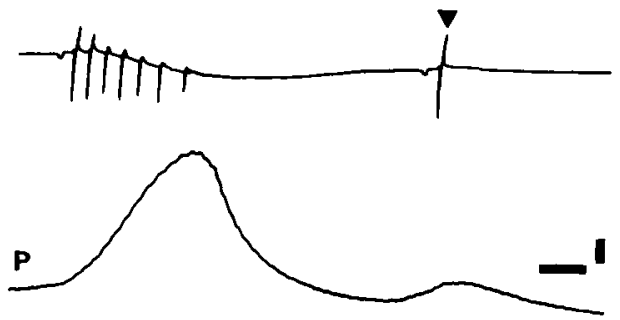

FIG. 6. - Enregistrement de l'activité du myomètre chez une brebis castrèe, anesthésiée 24 heures après l'injection de benzoate d'cestradiol.

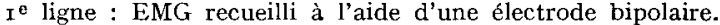

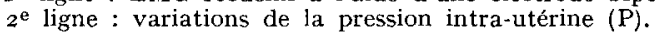

La salve est contemporaine de l'augmentation de la pression intra-utérine. En dehors de cette salve, à I potentiel d'action (flèche) correspond une faible variation de pression.

$$
\begin{array}{r}
\text { Étalonnages : amplitude : roo } \mu \mathrm{V}, 5 \mathrm{~mm} \mathrm{Hg}(\mathrm{P}) \\
\text { temps : } 2 \mathrm{~s}
\end{array}
$$


2. Action de la progestérone.

Chez la brebis castrée, anesthésiée 24 heures après la dernière injection d'un traitement de 6 jours à la progestérone, 1'activité EMG de 1'utérus est constituée par des variations lentes de potentiel durant Io à I5 secondes, plus ou moins différenciées par les constantes de temps utilisées ( 0,3 à $3 \mathrm{~s})$. Aucun potentiel d'action n'est par contre enregistré (fig. 5 B). Ces variations de potentiel ne sont jamais synchronisées avec les contractions du myomètre, lesquelles sont beaucoup plus faibles (3 à $5 \mathrm{~mm} \mathrm{Hg}$ ) et plus fréquentes ( 5 par $\mathrm{mn}$ ) que chez la brebis castrée sous imprégnation œstrogénique (fig. 5 A-B).

\section{B. - Étude chez l'animal castré éveillé}

Par rapport aux résultats précédents, les enregistrements chez l'animal éveillé permettent de suivre l'évolution de l'activité EMG du myomètre avant, pendant et après les traitements hormonaux.

Sur la brebis castrée éveillée, nous enregistrons avant tout traitement hormonal, une faible activité EMG qui se manifeste par périodes de 5 à Io minutes, 3 à 4 fois par heure. La figure $7 \mathrm{~A}$ montre une de ces phases actives, caractérisée par une succession de salves de faible amplitude ( silencieuse de durée équivalente (fig. 7 B).
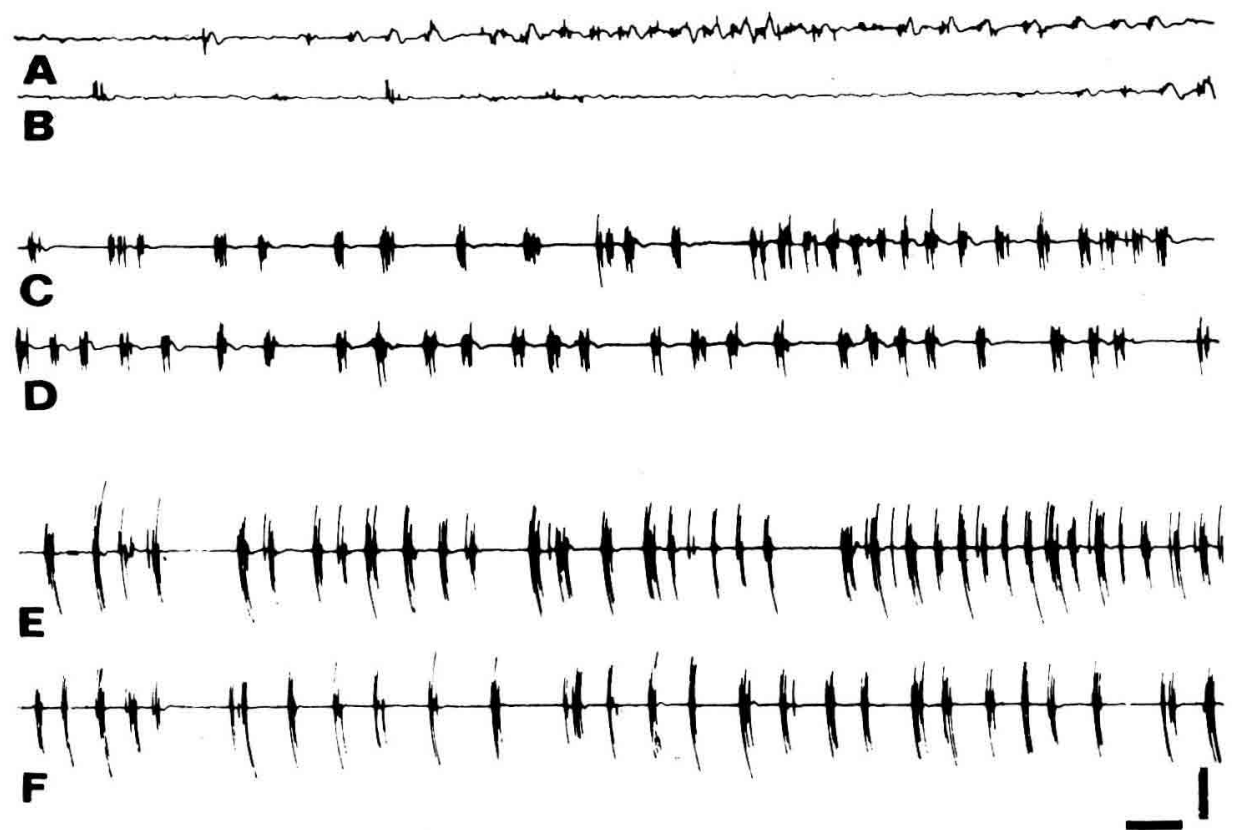

FIG. 7. - Enregistrement de l'activité du myomètre chez une brebis castrée éveillée

Les tracés $A$ et $B$ on ́́ été obtenus avant l'injection de benzoate d'œstradiol, $C$ et $D$ I 2 heures après, $E$ et F 24 heures après l'injection de benzoate d'ostradiol.

La fréquence des salves, ainsi que l'amplitude des potentiels d'action augmentent avec le temps. Les tracés $A$ et $B, C$ et $D, E$ et $F$ se font suite sans recouvrement.

$$
\begin{gathered}
\text { Étalonnages : amplitude : I mV } \\
\text { temps : } 40 \mathrm{~s}
\end{gathered}
$$


I. Action des cestrogènes.

Six à huit heures après l'injection d'œstrogènes, soit une quinzaine d'heures avant les premières manifestations comportementales de 1'œstrus inđuit, ces périodes silencieuses tendent à disparaître. Douze heures après l'injection, nous enregistrons, au rythme de 2,5 à 3 par minute, des salves de 4 à 5 potentiels dont l'amplitude est inférieure à $\mathrm{I} \mathrm{mV}$ (fig. $7 \mathrm{C}$-D). Au cours des heures suivantes, on assiste à une augmentation de la fréquence des salves qui devient maximale 24 à 36 heures après le traitement, au début de l'œstrus induit. Nous enregistrons alors par minute 3 à 4 salves composées de 4 à 8 potentiels dont la fréquence est d'environ un par seconde et dont 1'amplitude atteint I,2 à $2,5 \mathrm{mV}$ (fig. $4 \mathrm{~B}$ et fig. 7 E-F). Le second jour de l'œstrus, la fréquence des salves diminue, alors que les potentiels apparaissent à un rythme plus élevé, au sein de chaque salve (tabl. I), et nous ne recueillons plus qu'une bouffée de potentiels par minute 72 heures après l'injection, c'est-à-dire 48 heures environ après le début de l'œestrus (fig. $8 \mathrm{~A}$ ).
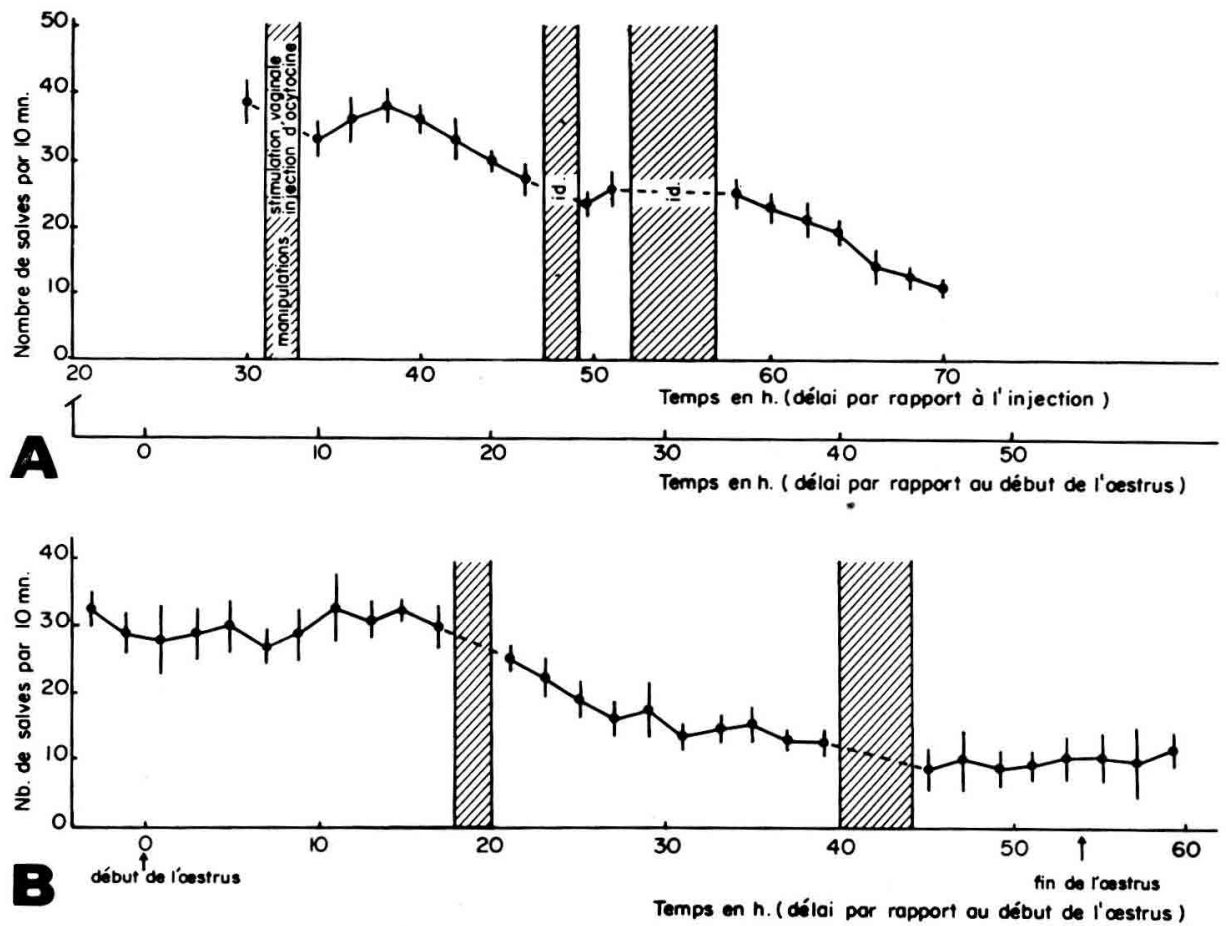

Fig. 8. - Évolution de la fréquence àes salves àe potentièls

chez une brebis castrée éveillée au cours d'un oestrus induit (A)

et chez une brebis cyclique éveillée au cours de l'oestrus (B)

Abscisses: en A : temps en heures par rapport au moment de l'injection d'œestrogènes et par rapport au début de l'œestrus induit.

en $\mathrm{B}$ : temps en heures par rapport au début de l'oestrus.

On a fait coincider le début de l'œstrus induit en A et celui de l'œstrus en B.

Ordonnées: en $\mathrm{A}$ et $\mathrm{B}:$ nombre moyen des salves de potentiels par Io $\mathrm{mn}$, déterminé par tranches successives de 2 heures. L'écart-type de part et d'autre de chaque point moyen est celui du nombre de salves comptées par périodes successives de Io $\mathrm{mn}$ pour chaque tranche de 2 heures.

Les zones en grisé correspondent aux modifications apportées par les stimulations vaginales et les injections d'ocytocine, dont nous n'avons pas tenu compte ainsi qu'aux périodes défectueuses d'enregistrement au cours desquelles les salves ne pouvaient pas être comotées. 


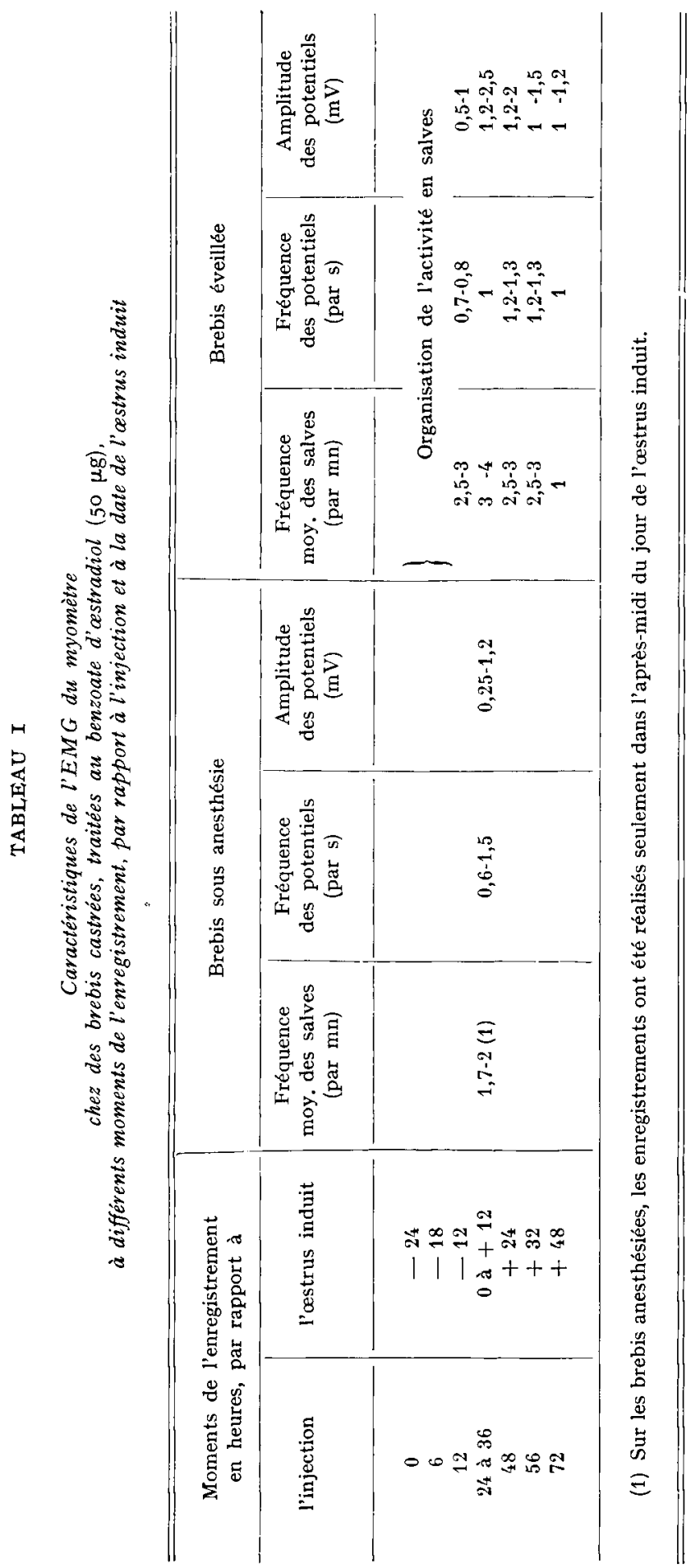




\section{Action de l'ocytocine.}

Des doses de 50 à $200 \mathrm{mU}$ d'ocytocine, injectée par voie intraveineuse chez une brebis castrée sous imprégnation œstrogénique provoquent, après un délai de 20 secondes, une augmentation de la fréquence des salves, telle que la décharge des potentiels d'action apparaît continue (fig. $9 \mathrm{~A}$ ). L'amplitude des potentiels, de $\mathrm{I}, 2$ à $2,5 \mathrm{mV}$, et leur fréquence, de $\mathrm{I}, 3$ par seconde, ne sont guère accrues par rapport aux caractéristiques des potentiels observés avant l'injection. L'augmentation de 1'activité électrique apparaît donc essentiellement due à une fréquence plus élevée des salves de potentiels. Elle dure environ 5 minutes après une injection de 50 à $100 \mathrm{mU}$, Io minutes après une injection de $200 \mathrm{mU}$. Des doses élevées de l'ordre de Io U d'ocytocine font disparaître les salves caractéristiques de l'activité du myomètre sous imprégnation œstrogénique.
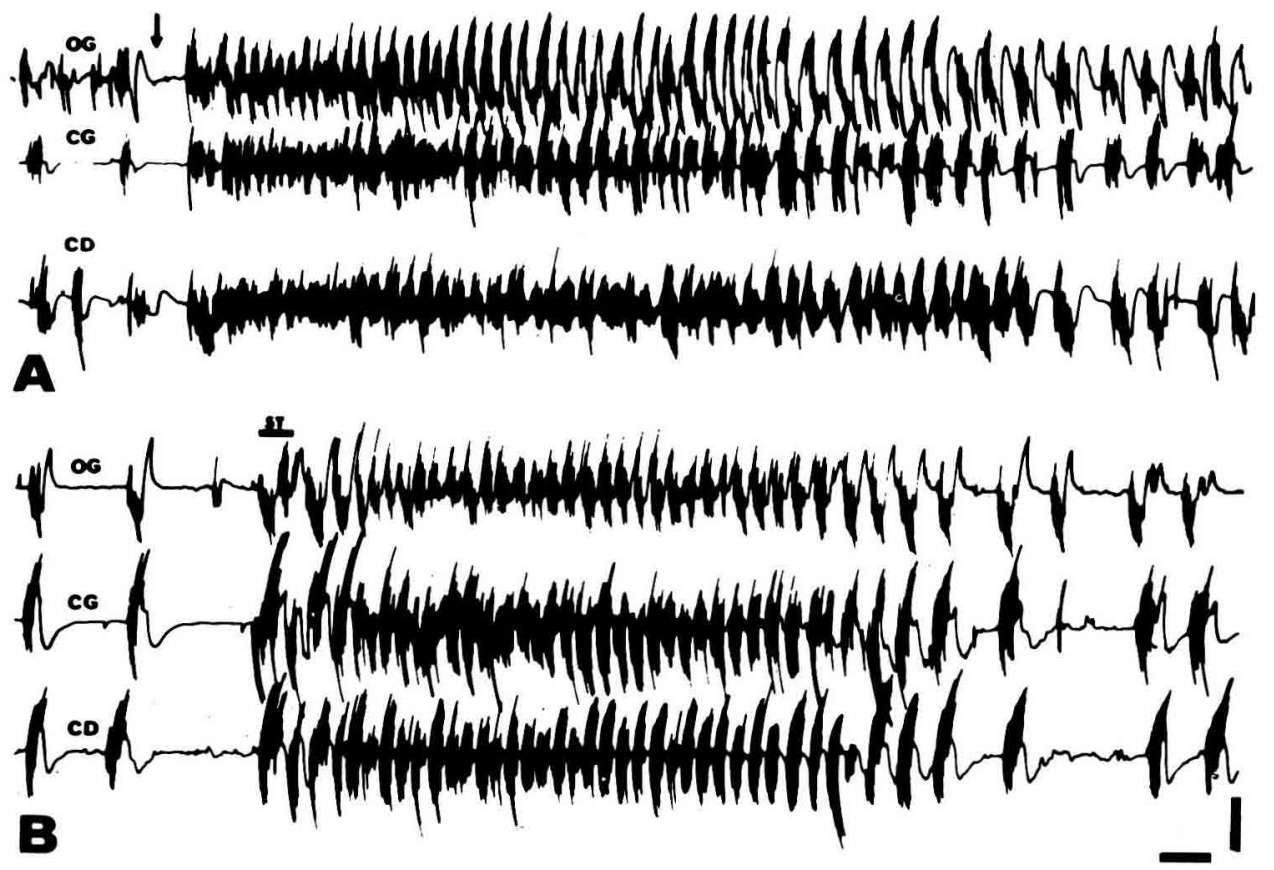

Fig. 9. - Activité électrique du myomètre enregistrée chez une brebis castrée, éveillée, en aestrus induit

EMG de la corne utérine gauche : $O G$ en région ovarienne, $\mathrm{CG}$ en région cervicale EMG de la corne utérine droite : $\mathrm{CD}$ en région cervicale

A : L'injection intraveineuse de roo $\mathrm{mU}$ d'ocytocine (indiquée par la flèche) provoque après un délai de I5 à 20 secondes, une augmentation importante de l'activité.

B : La distension brève du vagin (ST) produit un effet comparable qui se prolonge bien au-delà de la fin de la stimulation.

$$
\begin{aligned}
\text { Étalonnages : amplitude : } 2 \mathrm{mV} \\
\text { temps : } 30 \mathrm{~s}
\end{aligned}
$$

\section{Action de la progestérone.}

Les modifications de l'EMG sont enregistrées quatre heures après la première injection de progestérone : les potentiels d'action qui, avant traitement, étaient groupés par salves au sein de périodes actives, diminuent d'amplitude (50 à Ioo $\mu \mathrm{V})$. 
Ils apparaissent irrégulièrement au rythme d'un toutes les 2 à 20 secondes, greffés sur les variations lentes de potentiel, de 5 à Io secondes de durée, identiques à celles observées sur l'animal traité à la progestérone et anesthésié.

Ce type d'activité se poursuit pendant tout le traitement hormonal et persiste au cours des 24 heures qui suivent la dernière injection de progestérone,

\section{C. - Activité électrique du myomètre chez la brebis éveillée au cours du cycle oestrien}

Au cours de l'œstrus, nous enregistrons en un point de la corne utérine des salves de potentiels d'action identiques à celles observées chez la brebis castrée en œstrus induit. Pendant les 15 premières heures, la fréquence des salves est en moyenne de 3 par minute. Ces bouffées d'activité, de 5 à I 2 secondes de durée, sont constituées de 7 à 15 potentiels dont 1 'amplitude est de I à I, $2 \mathrm{mV}$ et dont la fréquence moyenne est de I potentiel par seconde. Elles sont synchrones de puissantes contractions d'environ 35 à $40 \mathrm{~mm}$ de $\mathrm{Hg}$ d'amplitude (fig. Io A). La fréquence des salves décroît

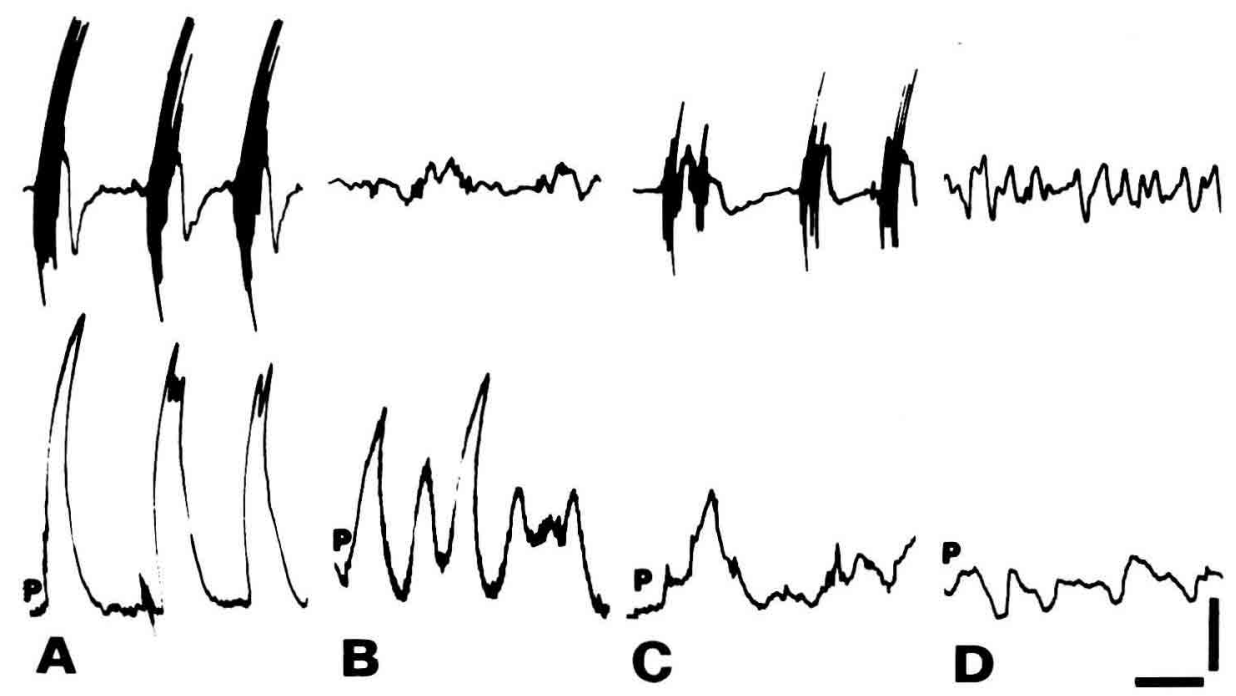

FIG. Io. - Enregistrement de l'activité du myomètre chez une brebis cyclique éveillée, à différents moments du cycle I $^{\text {re ligne }: E M G}$

$2^{\text {e }}$ ligne : variations de la tension musculaire $(P)$ recueillies au niveau d'un micro-ballonnet placé à l'intérieur du muscle utérin

A : Activité rythmique enregistrée le $1^{\text {er }}$ jour $d u$ cycle, soit 15 heures après le début de l'œstrus comportemental (à comparer avec la figure $5 \mathrm{~A}$ ).

$\mathrm{B}$ : Activité enregistrée le $4^{\mathrm{c}}$ jour du cycle.

$\mathrm{C}$ : Activité enregistrée le 6 e jour du cycle.

$\mathrm{D}:$ Activité enregistrée le $\mathrm{I}^{\mathrm{e}}$ jour du cycle (à comparer avec la figure $5 \mathrm{~B}$ ).

Étalonnages : amplitude : $300 \mu \mathrm{V}$, Io $\mathrm{mm} \mathrm{Hg}(\mathrm{P})$ temps: $20 \mathrm{~s}$

dès le premier jour de l'œstrus comportemental (fig. 8 B), alors que celle des potentiels au sein de chaque salve continue à augmenter pendant les $I 2$ heures suivantes, en même temps que l'amplitude de chaque contraction (tabl. 2). A la fin de l'œstrus, 
nous enregistrons seulement une salve par minute concomitante d'une contraction plus faible, de 20 à $30 \mathrm{~mm}$ de $\mathrm{Hg}$.

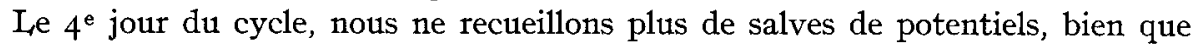
subsistent des contractions fortes (20 à $30 \mathrm{~mm}$ de $\mathrm{Hg}$ ) et fréquentes (4 par minute). La forme des contractions est modifiée : le mécanogramme présente, tant au cours des phases de contraction que des phases de relâchement, une suite d'épaulements qui n'apparaisssent pas sur les enregistrements mécaniques de l'ostrus (fig. Io B).

Chez quelques brebis, nous enregistrons à nouveau des salves au rythme de 2 à 2,5 par minute le $6^{\mathrm{e}}$ jour du cycle. L'amplitude des potentiels qui les composent est plus faible, de 0,5 à $0,6 \mathrm{mV}$. Leur fréquence est de I potentiel par seconde. Les contractions sont irrégulières, d'une amplitude variant de 2,5 à $15 \mathrm{~mm}$ de $\mathrm{Hg}$ (fig. Io C).

Des variations lentes de potentiel, de 5 à Io secondes de durée, sont recueillies tardivement du $g^{e}$ au $r 5^{e}$ jour du cycle. Elles ne sont pas synchrones de l'activité mécanique qui est caractérisée par des contractions fréquentes de faible amplitude

TABIEAU 2

Caractéristiques de l'EMG du myomètre chez la Brebis cyclique, au cours de l'cestrus

\begin{tabular}{c|c|c|c|c}
\hline \hline $\begin{array}{c}\text { Moment } \\
\text { de l'enregistrement } \\
\text { par rapport à la date } \\
\text { de l'œestrus (en h) }\end{array}$ & $\begin{array}{c}\text { Fréquence } \\
\text { moyenne } \\
\text { des salves } \\
\text { (par mn) }\end{array}$ & $\begin{array}{c}\text { Fréquence } \\
\text { des potentiels } \\
\text { (par s) }\end{array}$ & $\begin{array}{c}\text { Amplitude } \\
\text { des potentiels } \\
\text { (mV) }\end{array}$ & $\begin{array}{c}\text { Amplitude } \\
\text { des contractions } \\
\text { (mm de Hg) }\end{array}$ \\
\hline-16 & irrégulière & 0,9 & 0,25 & $5-15$ \\
-9 & 3 & 1 & $0,9-1$ & $25-40$ \\
+0 & 3 & 1 & $1-1,2$ & $35-40$ \\
+4 & 3 & 1,1 & $1-1,2$ & $35-40$ \\
+12 & 3 & 1,1 & $1-1,2$ & $35-40$ \\
+24 & 2 & 1,3 & $1-1,2$ & $40-50$ \\
+29 & $1,5-2$ & 1,4 & $1,-1,2$ & $40-50$ \\
+36 & $1-2$ & 1,4 & $0,8-0,9$ & $40-50$ \\
+48 & 1 & 1 & $0,5-0,6$ & $30-35$ \\
+57 & 1 & $0,3-0,4$ & $20-30$ \\
\hline \hline
\end{tabular}

( $5 \mathrm{~mm} \mathrm{de} \mathrm{Hg}$ ) (fig. Io $\mathrm{D}$ ). Ce type d'activité disparaît le $\mathrm{I} 6^{\mathrm{e}}$ jour du cycle où nous enregistrons à nouveau, mais irrégulièrement, des salves de potentiels du même type que celles observées durant l'œstrus. Une dizaine d'heures avant la détection de l'œstrus du cycle suivant, la fréquence de ces salves, 3 par minute, est déjà maximale (tabl. 2).

\section{D. - Modifications de l'activité électrique du myomètre au cours de stimulations vaginales chez la brebis éveillée}

Nous avons étudié les effets de deux types de stimulations vaginales sur la motricité de l'utérus sous imprégnation œstrogénique. On réalise soit une brève distension 
du vagin par un spéculum ou un ballonnet pendant ro à 20 secondes, soit une stimulation superficielle du vagin à 1'aide d'un écouvillon.

La distension provoque, chez la brebis éveillée en ostrus induit, une nette augmentation de l'activité électrique : 5 à 7 secondes après le début de la stimulation, la fréquence des salves croît de 2 à 6 par minute et se maintient à ce niveau pendant 5 minutes (fig. $9 \mathrm{~B}$ ). Par contre, l'amplitude des potentiels ( $\mathrm{I}$ à $2 \mathrm{mV}$ ) et leur fréquence (I par seconde) n'apparaissent pas augmentées. Un effet identique, bien que légèrement moins important, est obtenu chez la brebis cyclique en début d'œestrus. Dans les deux cas, l'augmentation d'activité persiste de 2 à 5 minutes après la fin de la brève distension.

La stimulation superficielle du vagin entrâne également une augmentation de la fréquence des salves qui croît de 2 à 4 par minute, sans toutefois produire de variations nettes de l'amplitude et de la fréquence des potentiels d'action, l'effet ne
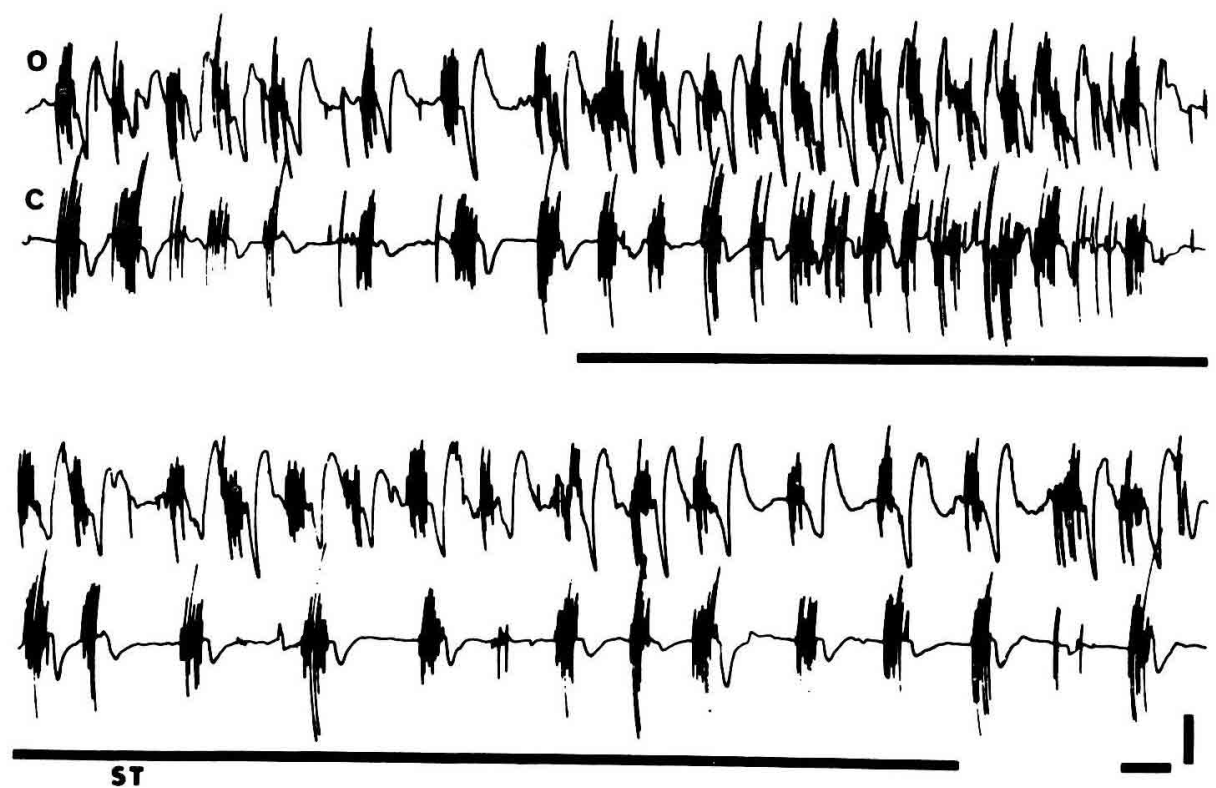

FIG. II. - Enregistrement de l'activité électrique du myomètre chez une brebis castrée éveillée, en oestrus induit

EMG d'une corne utérine : $O$ en région ovarienne, $C$ en région cervicale

La stimulation superficielle du vagin entraîne une augmentation de la fréquence des salves, qui cesse avant même la fin de la stimulation.

La durée de la stimulation est représentée par le trait horizontal (ST) situé au-dessous des enregistrements $\mathrm{O}$ et $\mathrm{C}$.

Étalonnages : amplitude : I $\mathrm{mV}$
temps : $\mathrm{I}_{5} \mathrm{~s}$

se prolonge pas au-delà de 4 à 6 minutes et cesse avant même l'arrêt de la stimulation (fig. II).

Enfin, la distension et la stimulation superficielle du vagin n'entraînent aucune modification de 1'activité électrique du myomètre chez les animaux anesthésiés. 


\section{DISCUSSION}

\section{A. - Influence des hormones sur l'activité du myomètre}

Les types d'activité électrique que nous avons recueillis in vivo chez la Brebis peuvent s'interpréter à la lumière des enregistrements intracellulaires réalisés in vitro, chez la Ratte et la Lapine par MARshali (I959), Kuriyana et Csapo (I96r), Csapo (I962), Marshal I (I962), Jung (I965). Ces auteurs montrent que les œstrogènes augmentent le potentiel de membrane à un niveau suffisant pour qu'apparaissent des potentiels d'action à la fréquence de 2 par seconde, groupés en trains qui précèdent et accompagnent chaque contraction. La progestérone a par contre, un effet hyperpolarisant sur le potentiel de membrane; les potentiels d'action sont irréguliers, d'amplitude variable, et leur décharge dans une fibre ne présente aucune relation constante avec la contraction du muscle.

Or, si nous comparons la taille des électrodes métalliques que nous utilisons à celle des fibres musculaires du myomètre (Io $\mu \mathrm{m} \times 300 \mu \mathrm{m})$ (CSAPO, Ig62), il est évident que l'électrode réceptrice doit recueillir les activités d'un grand nombre de générateurs. La forme simple de chaque potentiel (fig. 2 et 3) au sein de l'utérus sous imprégnation cestrogénique traduit donc l'activation synchrone d'un grand nombre de fibres. L'augmentation progressive de l'amplitude des potentiels au cours des 24 premières heures de l'œestrus signifierait alors que le nombre de fibres activées en phase croît pendant cette période. Cette activité synchrone semble pouvoir s'expliquer par l'existence de ponts intercellulaires de faible résistance (nexus) au niveau desquels s'effectue une transmission éphaptique de l'excitation; ces jonctions apparaissent d'ailleurs en grand nombre dans le myomètre de rattes castrées traitées préalablement par les œstrogènes, alors qu'on ne les observe pas chez les animaux témoins (BERGMAN, Ig68).

La mise en jeu synchrone d'un grand nombre de fibres permet d'expliquer les contractions rythmiques puissantes enregistrées au cours de l'œstrus. En effet, à une salve correspond toujours une contraction du myomètre. Nous retrouvons ainsi les résultats obtenus par MARSHaLL (I959), KuRIYAMa (I96I) sur des lambeaux du myomètre in vitro, par Bass et CaLLAN'TINe (1964) sur l'utérus in vivo, mais les rapports chronologiques entre les activités électrique et mécanique ne sont pas aussi constants que le montrent les enregistrements de MARshal, (I959). Nous avons remarqué que souvent même le début de la contraction précède l'activité EMG. Ceci est vraisemblablement dû aux conditions d'enregistrement de l'activité mécanique. Lorsqu'on recueille les variations de la pression intra-utérine à l'aide d'un ballonnet gonflé d'air, celui-ci détecte la variation globale de la pression qui s'exerce à l'intérieur de toute la corne utérine, alors qu'on enregistre une activité EMG très localisée. De plus, sous l'action de la motricité utérine, le ballonnet se déplace légèrement par rapport aux électrodes qui, elles, restent fixes. Par contre, le début de la salve de potentiels précède toujours celui de la contraction, d'une demi-seconde environ, lorsque nous recueillons l'activité mécanique grâce à un micro-ballonnet inséré dans la paroi musculaire, au voisinage des électrodes de réception. 
Par suite de l'effet hyperpolarisant de la progestérone, les fibres musculaires sont moins excitables et leur potentiel de membrane est plus stable que sous imprégnation œstrogénique. Les potentiels d'action ne sont pas propagés (MARSHALL, I959, I962). Aussi, les cellules musculaires ne déchargent-elles pas en phase, ce qui expliquerait la disparition des salves et les variations lentes de potentiel que nous enregistrons chez les brebis castrées traitées à la progestérone. Les variations de la pression intra-utérine seraient alors le résultat de la coalescence de nombreuses et faibles contractions localisées, comme on peut l'observer sur la figure ro B.

L'action de l'ocytocine que nous avons constatée sur l'utérus sous imprégnation œstrogénique s'explique par les résultats obtenus sur la fibre lisse utérine in vitro par Kuriyama et Csapo (I96I) et par Marshali, (I962) : des faibles doses dépolarisent la membrane jusqu'au seuil critique nécessaire pour qu'apparaisse le potentiel d'action, tandis que des fortes doses entraînent une dépolarisation soutenue de la membrane; cette dépolarisation excessive ne permet plus la naissance de potentiels d'action. Cela se traduit au niveau de nos enregistrements globaux par une disparition des salves qui peut laisser croire à une inhibition de la motricité utérine, alors que le muscle est en état de contracture.

\section{B. - Modifications de l'activité utérine au cours du cycle oestrien}

L'activité du myomètre enregistrée chez la Brebis cyclique dès la fin du cycle et pendant l'œstrus suivant est identique à celle observée chez la Brebis castrée sous imprégnation œstrogénique. Il en est de même pour l'évolution de cette activité en fonction du temps. Or, le niveau d'œstradiol libéré dans le sang est maximal 40 heures avant le début de l'œstrus, et il diminue pour être indécelable 24 heures après le début de l'œstrus (Moore et al., r969). Chez la femelle cyclique, l'activité EMG rythmique spontanée qui caractérise l'œstrus est donc le résultat d'une forte imprégnation par les ostrogènes, et il faut un délai d'environ $\mathrm{I} 2$ heures après ce pic pour qu'apparaissent les premières modifications caractéristiques de l'activité électrique, avant même que l'œstrus comportemental ne soit détecté.

De la même façon, les salves que nous avons recueillies le $6^{\text {e jour }} \mathrm{du}$ cycle chez quelques brebis (fig. Io C) sont vraisemblablement liées à une $2^{\mathrm{e}}$ sécrétion d'œstrogènes. Celle-ci est notée le $3^{\mathrm{e}}$ et le $4^{\mathrm{e}}$ jour du cycle par Cox, MatTner et ThorBURN (I97I), le $6^{\mathrm{e}}$ et le $7^{\mathrm{e}}$ jour par OBST, SEAMARK et Brown (I97I). Une élévation du niveau d'œstrogènes le $2^{\mathrm{e}}$ et le $8 \mathrm{e}$ jour du cycle est aussi observée par Scaramuzzi, CALDWELl et MOORE (I970). Si l'existence de cette $2^{\text {e }}$ sécrétion semble bien établie par ces auteurs, sa place dans le cycle et son amplitude semblent variables. L,e niveau de progestérone s'élève par ailleurs à partir du $2^{\mathrm{e}}$ jour du cycle. Le $6^{\mathrm{e}}$ jour du cycle, il atteint déjà la moitié du niveau maximal (ThIMONIER, communication personnelle). Nous pouvons donc supposer que le rapport œestrogènes/progestérone au moment de cette $2^{\text {e }}$ sécrétion d'œstrogènes varie d'une brebis à l'autre. Dans ces conditions, 1'activité du myomètre que nous recueillons à cette époque dépendra de ces variations.

Nous retrouvons du $9^{\mathrm{e}}$ au $\mathrm{I}^{\mathrm{e}}$ jour du cycle le même type d'activité que celui que nous enregistrons chez la Brebis castrée sous imprégnation de progestérone. Or, nous savons qu'à cette période du cycle le niveau de progestérone dans le sang est 
élevé : la progestéronémie est maximale entre le $\mathrm{II} \mathrm{I}^{\mathrm{e}}$ et le $\mathrm{I} 2^{\mathrm{e}}$ jour du cycle et elle diminue rapidement le $\mathrm{I}_{4}^{\mathrm{e}}$ jour du cycle (MOORE et al., I969; THIMONIER, communication personnelle).

Il apparaît donc que l'on peut expliquer les modifications de l'activité électrique du myomètre par les variations des teneurs plasmatiques en hormones sexuelles au cours du cycle.

\section{C. - Influence de l'anesthésie sur l'activité du myomètre}

La comparaison des résultats obtenus, sous imprégnation d'œstrogènes, sur les brebis castrées, anesthésiées ou éveillées, nous permet de discuter le rôle de l'anesthésie sur la motricité utérine. L'activité du myomètre est en effet déprimée par le fluothane comme le montre la figure 4. Comparé au même moment de l'œestrus induit, 24 heures après l'injection d'œestrogènes, le rythme des contractions est deux fois plus lent chez la Brebis anesthésiée que chez la Brebis éveillée (fig. $4 \mathrm{~A}-\mathrm{B}$ ). Ce fait a été observé chez la Femme (VASICKA et KRETCHMER, I96I ; MILLER et al., Ig66) et chez la Lapine (ZAUDER et al., I970) in vivo au cours de l'accouchement, in vitro sur des lambeaux d'utérus prélevés lors du part. Il est vraisemblable que la fibre musculaire elle-même est sensible à l'action du fluothane. Ce problème est important, car on déprime l'activité du myomètre lors des accouchements dystociques pratiqués sous anesthésie, mais cette action du fluothane peut être intéressante en obstétrique lorsqu'un relâchement utérin est nécessaire (CRAWFORD, I962).

\section{D. - Influence de stimulations périphériques vaginales sur l'activité du myomètre}

On admet généralement qu'une stimulation génitale provoque la décharge d'hormones post-hypophysaires (VANDEMARK et Hays, I952; Debackere et al., I96r). Chez la Brebis, Richard (I969) recueille dans la tige pituitaire des potentiels évoqués par des stimulations vaginales, dont l'apparition précède l'augmentation de la pression intramammaire, et qui sont vraisemblablement en rapport avec une libération d'ocytocine. Nous avons donc comparé l'activité provoquée par des stimulations vaginales à celle observée après injection d'ocytocine. La figure 9 montre que la distension vaginale chez la Brebis castrée en cestrus induit détermine une augmentation de l'activité électrique comparable à celle obtenue après une injection de $0, \mathrm{I}$ UI d'ocytocine. La réponse de longue durée à une brève distension vaginale fait d'ailleurs penser à la mise en jeu d'un réflexe neuro-hormonal plutôt qu'à celle d'un réflexe purement nerveux. Elle est cependant de plus courte latence (5 à 7 secondes) que la réponse du myomètre à l'injection d'ocytocine (I5 à 20 secondes). Une latence de même ordre est d'ailleurs retrouvée par LIGHTFoor (I970), après une injection d'ocytocine chez la Brebis en ostrus, alors que l'intromission du pénis détermine une augmentation immédiate de la motricité utérine. On peut émettre 1'hypothèse que l'accroissement de l'activité, lors d'une brève distension, est le résultat de la mise en jeu d'un réflexe nerveux court, spinal ou pontique et qu'un réflexe long neuro-endrocrinien, hypothalamo-hypophysaire, prendrait le relais, afin d'assurer 1'hyperactivité du myomètre bien après l'arrêt de la stimulation. L'effet de la stimu- 
lation superficielle, moins important que celui de la distension vaginale, et qui cesse avant même l'arrêt de la stimulation, résulterait par contre de la seule mise en jeu d'un réflexe nerveux court.

Reçu pour publication en juin 1973.

\section{SUMMARY}

\section{ELECTROMYOGRAPHIC STUDY OF UTERINE ACTIVITY IN THE EWE}

We have recorded in vivo the electrical and mechanical activity of the myometrium in 46 ewes including cyclic animals, and ovariectomized animals treated with œestrogen or progesterone.

Electrical activity was recorded in anaesthetized animals using either extracellular glass $\mathrm{KCl}$ electrodes, or external metallic electrodes fixed onto the serous. In conscious animals, platinum electrodes were implanted permanently in the uterus. Mechanical activity was evaluated from variations in intrauterine pressure or local variations in muscular tension.

The results showed that :

I. In the ovariectomized ewe.

- Estrogen induces rythmic electromyographic activity (EMG) composed of bursts of action potentials related to the variation in intra-uterine pressure. This EMG activity is at a maximum 24 to $3^{6}$ hours after treatment with ostradiol benzoate, which coincide with the beginning of œestrus.

- After treatment with progesterone, uterine EMG activity is composed of slower variations in potential, which are not related to myometrial contractions.

- After cestrogen treatment, low doses of oxytocin provoke a continuous discharge of action potentials whereas high doses abolish them.

2. In cyclic ewes during œstrus, the uterus exhibits a spontaneous rythmic EMG activity, indentical with that induced by estrogen in the ovariectomized animal. Except for a few ewes which showed spontaneous activity on day 6 of the oestrous cycle, this activity, characterised by bursts of action potentials, seems to be confined to the last day of the cycle and the first 3 days of the following one.

3. In the conscious ewe, whether ovariectomized and cestrogen-treated, or intact at the beginning of cestrus, vaginal stimulation (distension or superficial stimulation of the vagina) induces a marked increase in electrical activity of the myometrium. This same stimulation has no effect on the anaesthetized animal.

\section{RÉFÉRENCES BIBLIOGRAPHIQUES}

Auffray P., Martinet J., Rerat A., I967. Quelques aspects du transit gastro-intestinal chez le Porc. Ann. Biol. anim. Biochim. Biophys., 7, 26r-279.

Bass P., Callantine M. R., 1964. Simultaneous recording of electrical and mechanical activity of the uterus in the unanaesthetized animal. Nature, Lond., 203, 1367-I368.

Bergman R. A., r968. Uterine smooth muscle fibres in castrate and oestrogen treated rats. J. Cell. Biol., 36, 639-648.

Brinsfield T. H., I968. Relationship of endocrine state to spontaneous motility of sheep myometrium in vitro. J. Anim. Sci., 27, 439-442.

Cox R. I., Mattner P. E., Thorburn C. D., I97r. Changes in ovarian secretion of cestradiol I7 $\beta$ around œstrus in the sheep. J. Endocr., 49, 345-346.

Crawford J. S., 1962. The place of halothane in obstetrics. Br. J. Anaesth., 34, 386-39o.

Csapo A. I., rg62. Smooth muscle as a contractile unit. Physiol. Rev., 42 (suppl. 5), 7-33.

Csapo A. I., TAKEdA A., 1963. Electrical activity of the parturient human uterus. Nature, Lond., $200,680-682$. 
Debackere M., Peeters G., Tuyttens N., 196r. Reflex release of an oxytocic hormone by stimulation of genital organs in male and female sheep studied by a cross circulation technique. J. Endocr., 22, $32 \mathrm{I}-334$.

Effer S. B., Bertora R. P., Vrettos A., Caldeyro-Barcia R., ig69. Quantitative study of the regularity of uterine contractile rythm in labor. Amer. J. Obstet. Gynec., 105, 909-915.

Hindson J. C., Schofield B. M., Turner C. B., i968. Parturient pressures in the ovine uterus. J. Physiol., Lond., 195, 19-28.

Jung H., I965. Erregung-physiologie des Uterus. Arch. Gynaek, 202, I4-22.

Krapohl A. J., Myers G. G., Caldeyro-Barcia R., i97o. Uterine contractions in spontaneous labor. Amer. J. Obstet. Gynec., 106, 378-387.

Kuriyama H., I96r. Recent studies on the electrophysiology of the uterus. In Progesterone and the defence mechanism of pregnancy, Ciba Fdn Study Grp n ${ }^{\circ}$ 9, London : Churchill, $5 \mathrm{I}-7 \mathrm{I}$.

Kuriyama N., Csapo A., I961. A study of the parturient uterus with the micro-electrode technique. Endocrinology, 68, 1010-1025.

Lightfoor R. J., I97o. The contractile activity of the genital tract of the ewe in response to oxytocin and mating. J. Reprod. Fert., 21, 376.

MANN L. I., I969. Effect of the IUCD on uterine motility in the ewe. Fert. Steril., 20, 95I-958.

Marshall J. M., I959. Effects of œstrogen and progesterone on single uterine muscle fibers in the rat. Amer. J. Physiol., 197, 935-942.

Marshall J. M., I962. Regulation of activity in uterine smooth muscle. Physiol. Rev., 42 (suppl. 5), $213-227$.

Miller J. R., Stoelting V. K., stander R. W., Watring w., I966. In vitro and in vivo responses of the uterus to halothane anesthesia. Anesth. Analg. curr. Res., 45, 583-589.

Moore N. W., Barret S., Brown I. B., Schindler I., Smith M. A., Smyth B., r969. Estrogen and progesterone content of ovarian vein blood of the ewe during the ostrous cycle. $J$. Endocr., 44, 55-62.

Nastuk W. L., I963. Physical technique in biological research., Vol. VI, part B. New-York, Academic Press, $425 \mathrm{p}$.

Obst J. M., Seamark R. F., Brown J. M., i97I. Application of competitive protein binding assay for œstrogens to the study of ovarian function in sheep. J. Reprod. Fert., 24, 140.

RICHARD P., I969. Contribution à l'étude des voies nerveuses du réflexe neuroendocrinien d'éjection du lait chez la Brebis. Thèse Doct. Sci. Nat., Paris, 172 p.

Robinson T. J., I962. Comparative studies of several gonadotrophin, progestin and cestrogen treatments in the anœstrous ewe. J. Endocr., 24, 33-5 r.

Rousseau J. P., 1970. Contribution à l'êtude de la rumination et de l'éructation chez le Mouton. Thèse Doct. Sci. Nat., Marseille, I56 p.

Scaramuzzi R. J., Caldwell B. V., Moore R. M., r97o. Radioimmunoassay of LH and oestrogen during the estrous cycle of the ewe. Biol. Reprod., 3, I IO-II9.

Vandemark N. L., Hays R. L., 1952. Uterine motility responses to mating. Amer. J. Physiol., 170, 5I8-52I.

VASICKa A., KRetchmer H., I96I. Effect of conduction and inhalation anesthesia on uterine contractions. Amer. J. Obstet. Gynec., 82, 600-6Ir.

Wolfs G., Rottinghuis H., 1970. Electrical and mechanical activity of the human uterus during labor. Arch. Gynaek., 208, 373-385.

Woodbury J. W., Brady A. J., 1956. Intracellular recording from moving tissue with a flexibly mounted ultramicro-electrode. Science $N$. Y., 123, Ioo-ror.

Zauder H. L., Pauerstein C. J., Fremming B. D., Filner B., 197o. Failure of propanolol to antagonize halothane depression of the uterus. Anesth. A nalg. curr. Res., 49, 948-956. 